\title{
Thermal Conductivity of Monolayer Molybdenum Disulfide Obtained from Temperature-Dependent Raman Spectroscopy
}

\author{
Rusen Yan, ${ }^{+, *}$ Jeffrey R. Simpson, ${ }^{\ddagger}$ Simone Bertolazzi, ${ }^{\perp}$ Jacopo Brivio, ${ }^{\perp}$ Michael Watson, ${ }^{\ddagger}$ Xufei Wu," \\ Andras Kis, ${ }^{\perp}$ Tengfei Luo," Angela R. Hight Walker, ${ }^{\varsigma, *}$ and Huili Grace Xing ${ }^{\dagger, *}$
}

${ }^{\dagger}$ Department of Electrical Engineering, University of Notre Dame, Notre Dame, Indiana 46556, United States, ${ }^{\ddagger}$ Department of Physics, Astronomy, and Geosciences, Towson University, Towson, Maryland 21252, United States, ${ }^{8}$ Semiconductor and Dimensional Metrology Division, National Institute of Standards and Technology (NIST), Gaithersburg, Maryland 20899, United States, ${ }^{\perp}$ Electrical Engineering Institute, Ecole Polytechnique Federale de Lausanne (EPFL), CH-1015 Lausanne, Switzerland, and "Department of Aerospace and Mechanical Engineering, University of Notre Dame, Notre Dame, Indiana 46556, United States

\begin{abstract}
Atomically thin molybdenum disulfide $\left(\mathrm{MoS}_{2}\right)$ offers potential for advanced devices and an alternative to graphene due to its unique electronic and optical properties. The temperature-dependent Raman spectra of exfoliated, monolayer $\mathrm{MoS}_{2}$ in the range of $100-320 \mathrm{~K}$ are reported and analyzed. The linear temperature coefficients of the in-plane $E_{2 g}{ }^{1}$ and the out-of-plane $A_{1 g}$ modes for both suspended and substrate-supported monolayer $\mathrm{MoS}_{2}$ are measured. These data, when combined with the first-order coefficients from laser power-dependent studies, enable the thermal conductivity to be extracted. The resulting thermal conductivity $\kappa=(34.5 \pm 4) \mathrm{W} / \mathrm{mK}$ at room temperature agrees well with the firstprinciples lattice dynamics simulations. However, this value is significantly lower than that of graphene. The results from this work provide important input for the design of $\mathrm{MoS}_{2}$-based devices where thermal management is critical.
\end{abstract}

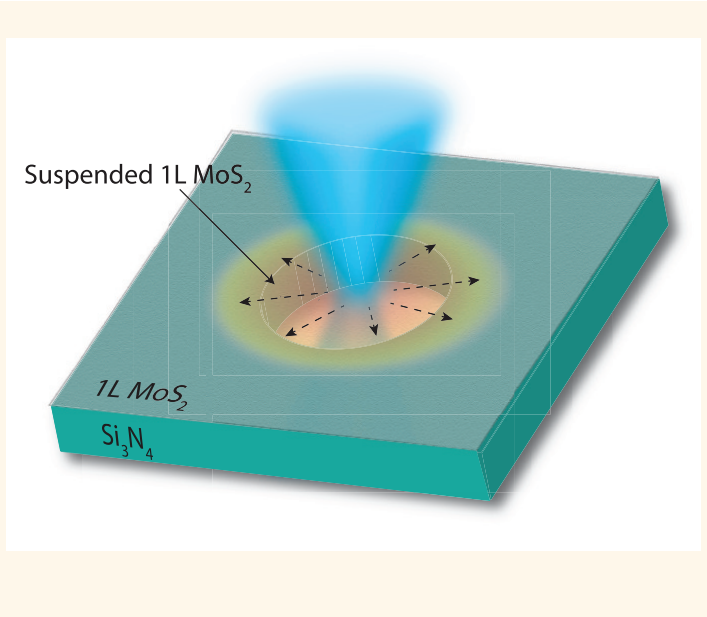

KEYWORDS: molybdenum disulfide $\cdot$ thermal conductivity $\cdot$ Raman spectroscopy $\cdot$ first-principle lattice dynamics $\cdot$ suspended

1 he discovery of graphene ${ }^{1-3}$ nearly a decade ago has stimulated intensive research efforts in atomically thin, two-dimensional (2D) crystals, such as transition-metal dichalcogenide (TMD) semiconductors $^{3-6}$ and boron-nitride (BN). ${ }^{4}$ Compared to graphene, the presence of a bandgap in TMDs is more desirable for device applications. Recent experimental and theoretical work shows that these layered TMDs undergo a transition from indirect to direct bandgap when their thickness is reduced from bulk to monolayer, leading to a pronounced photoluminescence $(\mathrm{PL}) .^{7-9}$ Among this family, molybdenum disulfide $\left(\mathrm{MoS}_{2}\right)$ is one of the most stable layered TMDs. Bulk $\mathrm{MoS}_{2}$ consists of S-Mo-S layers weakly bonded by van der Waals (vdW) interactions and has been widely used in commercial application as a dry lubricant.
Transistors constructed from few-layer and monolayer $\mathrm{MoS}_{2}$ show potential for future low-power device platforms. ${ }^{10,11}$ The indirect gap at $1.2 \mathrm{eV}$ in bulk $\mathrm{MoS}_{2}$ gradually evolves into a direct gap at $1.9 \mathrm{eV}$ in the monolayer form. As the layer number increases, the presence of interlayer vdW forces affects not only the band structure, $^{12,13}$ but also the lattice vibrations. ${ }^{14}$ As a result, Raman spectroscopy, which is especially sensitive to phonon frequency changes, is used to determine the layer number of 2D crystals including graphene, ${ }^{15}$ $\mathrm{WS}_{2}{ }^{16}$ and $\mathrm{MoS}_{2}{ }^{14}{ }^{14}$ Two prominent peaks, the in-plane $E_{2 g}^{1}$ and the out-of-plane $A_{1 g}$ modes, are observed in the Raman spectrum of $\mathrm{MoS}_{2}$. Moreover, the phonon frequency difference between the two modes is widely used as the thickness indicator. ${ }^{14}$ It has also been shown that Raman spectral

\begin{abstract}
* Address correspondence to ryan1@nd.edu, angela.hightwalker@nist.gov hxing@nd.edu.
\end{abstract}

Received for review November 10, 2013 and accepted December 30, 2013.

Published online December 30, 2013 $10.1021 / \mathrm{nn} 405826 \mathrm{k}$

() 2013 American Chemical Society 
(a)

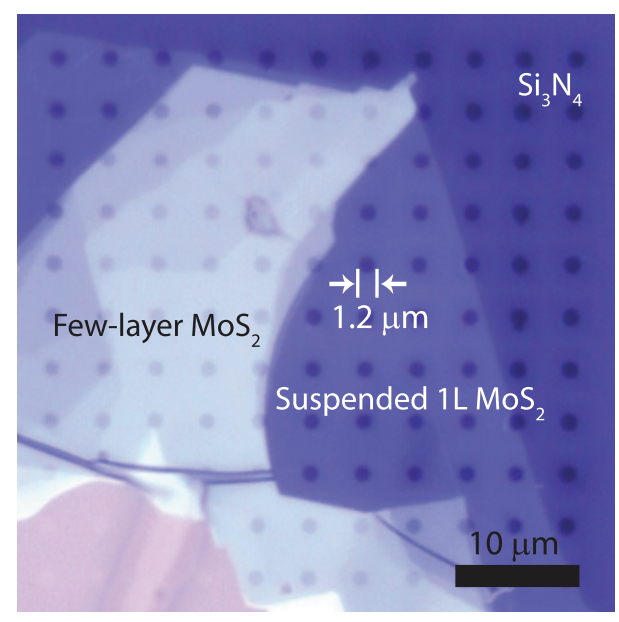

(b)

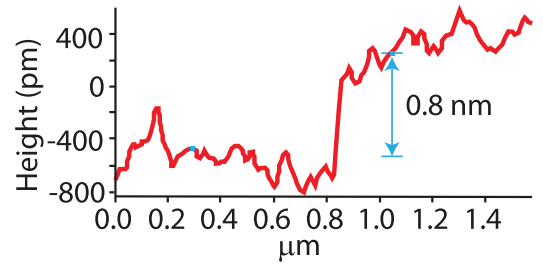

(c)

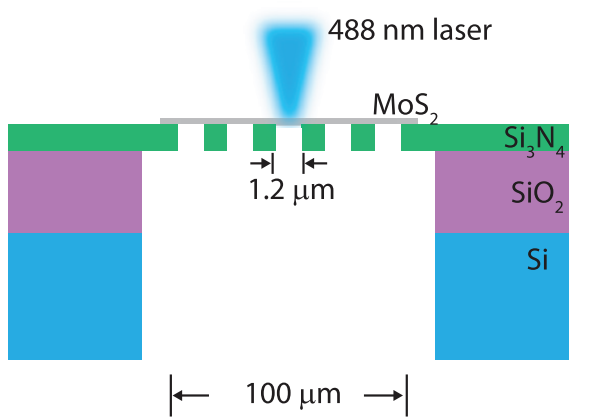

Figure 1. (a) Optical micrograph of exfoliated $\mathrm{MoS}_{2}$ flakes placed on $\mathrm{Si}_{3} \mathrm{~N}_{4} / \mathrm{SiO}_{2} / \mathrm{Si}$ perforated grid. (b) AFM step height across the edge of the monolayer $\mathrm{MoS}_{2}$ flake on PMMA/PVA prior to being transferred to the final substrate, and the roughness revealed by AFM largely stems from PMMA/PVA. (c) Sketch of monolayer $\mathrm{MoS}_{2}$ on $\mathrm{Si}_{3} \mathrm{~N}_{4} / \mathrm{SiO}_{2} / \mathrm{Si}$ substrate. Here MoS $\mathrm{M}_{2}$ over the holes in the $20 \mathrm{~nm}$ thick $\mathrm{Si}_{3} \mathrm{~N}_{4}$ is suspended.

features can be used to characterize doping, the dielectric environment, and strain effects of atomically thin $\mathrm{MoS}_{2}$ flakes by monitoring the change of peak position and full width at half-maximum (fwhm) of Raman-active phonons. ${ }^{17-20}$ In this article, we present a detailed temperature- and laser-power-dependent Raman study of monolayer $\mathrm{MoS}_{2}$. To isolate the substrate effects, we compare Raman measurements from both suspended and supported monolayer $\mathrm{MoS}_{2}$ flakes. Such studies are of fundamental importance for a comprehensive understanding of anharmonic lattice vibrations, thermal expansion, and thermal conductivity. $^{21-25}$ Our temperature-dependent Raman study can provide a powerful, noncontact method to monitor the local temperature rise induced by light, electrical currents, and so on. The combination of both temperature and power dependence of optical phonon frequencies permits extraction of the thermal conductivity $\kappa .^{26,27}$ The measured value and that calculated from first-principles lattice dynamics simulations show reasonable agreement. The relatively low thermal conductivity value of $\mathrm{MoS}_{2}$ should be carefully considered

\section{in} the design of $\mathrm{MoS}_{2}$ based electronics, optics, and thermoelectronics.

\section{RESULTS AND DISCUSSION}

The optical micrograph of suspended monolayer over a $20 \mathrm{~nm}$ thick, perforated $\mathrm{Si}_{3} \mathrm{~N}_{4}$ grid is shown in Figure 1(a). The height profile measured by AFM at the edge of the flake on rough PMMA/PVA surface before the transfer process, presented in Figure 1(b), shows a thickness of $\approx 0.8 \mathrm{~nm}$ with a root-mean-square roughness larger than $0.3 \mathrm{~nm}$, confirming that the region of interest is indeed monolayer $\mathrm{MoS}_{2}$. The cross-section of the $\mathrm{Si}_{3} \mathrm{~N}_{4} / \mathrm{SiO}_{2} / \mathrm{Si} \mathrm{substrate}$ structure is shown in Figure 1 (c), where holes with a diameter of $1.2 \mu \mathrm{m}$ are defined by e-beam lithography and patterned by an etching process on $\mathrm{Si}_{3} \mathrm{~N}_{4}$. The suspended monolayer $\mathrm{MoS}_{2}$ flake is obtained on top of the holes in $20 \mathrm{~nm}$ thick $\mathrm{Si}_{3} \mathrm{~N}_{4}$.

The temperature-dependent Raman measurements were collected using a Renishaw InVia micro-Raman spectrometer employing an excitation laser with wavelength of $514.5 \mathrm{~nm}$. A long-working distance $50 \times$ objective lens (numerical aperture NA $=0.55$ ) provides a Gaussian beam width estimated to be $2 \lambda_{0} /(\pi \mathrm{NA}) \approx 0.6 \mu \mathrm{m} .{ }^{28}$ The samples were mounted in a cryostat cooled by liquid nitrogen. Throughout all of the temperature-dependent measurements, the laser power is maintained to be less than $0.14 \mathrm{~mW}$.

Figure 2(a) shows four example Raman spectra and their corresponding Lorentzian fits collected from suspended monolayer $\mathrm{MoS}_{2}$ while the cryostat temperature ranges from 100 to $320 \mathrm{~K}$. As seen in the figure, two prominent peaks around 385 and $405 \mathrm{~cm}^{-1}$ are observed, which correspond to the in-plane $E_{2 g}^{1}$ mode and the out-of-plane $A_{1 g}$ mode, respectively. ${ }^{14}$ Vibration of the $E_{2 g}^{1}$ mode involves the in-plane opposing motions of sulfur ( $\mathrm{S}$ ) and molybdenum (Mo) atoms and that of $A_{1 g}$ mode is the out-of-plane relative motions of $S$ atoms, as depicted in the insets of Figure 2(a). As the temperature increases, both of the Raman-active modes soften linearly and the $A_{1 g}$ peak clearly broadens (see also Figure S1, Supporting Information). Figure 2(b) shows the temperature dependence of the Lorentzian-fit peak frequencies from 100 to $320 \mathrm{~K}$ for the $A_{1 g}$ (blue squares) and $E_{2 g}^{1}$ (red circles) phonons. The evolution of the Raman peak position $\omega$ (in $\mathrm{cm}^{-1}$ units) as a function of lattice temperature follows a linear dependence 


$$
\Delta \omega=\omega\left(T_{2}\right)-\omega\left(T_{1}\right)=\chi_{T}\left(T_{2}-T_{1}\right)=\chi_{T} \Delta T
$$

where $\chi_{T}$, the slope of temperature dependence, is the first-order temperature coefficient for the respective modes, and $T$ is the absolute temperature. Nonlinear coefficients are not considered here, given that they can only be observed at higher temperatures. ${ }^{26,27}$ It is worth noting that we observed nonlinear dependence in our laser power measurements at room temperature (RT), which will be discussed later in this paper. The extracted linear temperature coefficients $\chi_{T}$ from the slopes are $-(0.011 \pm$ $0.001) \mathrm{cm}^{-1} / \mathrm{K}$ and $-(0.013 \pm 0.001) \mathrm{cm}^{-1} / \mathrm{K}$ for the $E_{2 g}^{1}$ and $A_{1 g}$ modes, respectively. In the following, the

(a)

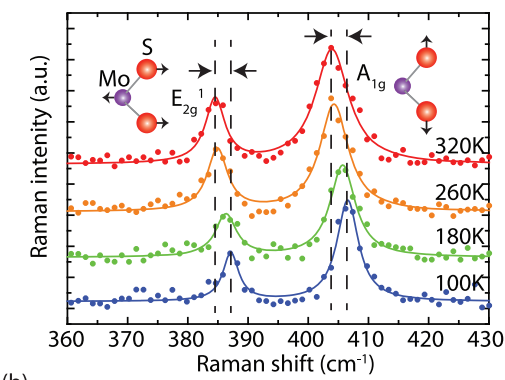

(b)

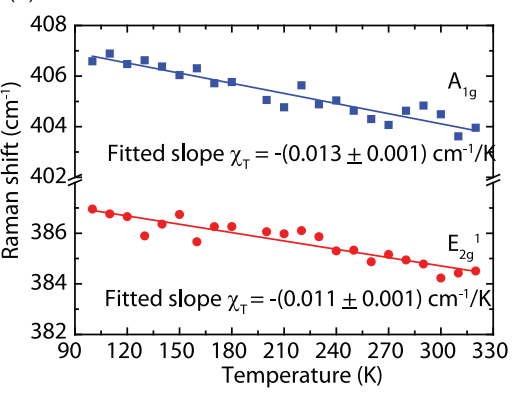

Figure 2. (a) Four example Raman spectra of suspended, monolayer $\mathrm{MoS}_{2}$ collected at 100, 180, 260, and $320 \mathrm{~K}$. Spectra offset vertically for clarity. (b) Raman peak frequencies of both $A_{1 g}$ (blue squares) and $E_{2 g}^{1}$ (red circles) modes as a function of temperature. Fit lines and resulting linear temperature coefficients $\chi_{T}$ are shown. value for $A_{1 g}$ mode is used to extract the thermal conductivity.

The observed linear evolution of phonon frequencies in monolayer $\mathrm{MoS}_{2}$ can be attributed to the anharmonic vibrations of the lattice, ${ }^{21}$ which mainly includes contributions from the lattice thermal expansion due to the anharmonicity of the interatomic potential. As the lattice expands or contracts because of temperature change, the equilibrium positions of atoms and consequently the interatomic forces change, which induces shifts in the phonon energies. ${ }^{29}$ This linear behavior of Raman peak frequencies with temperature is seen in many materials within a certain temperature range. ${ }^{22,23,29}$ In Table 1, the linear coefficients of suspended monolayer $\mathrm{MoS}_{2}$ (this work), suspended few-layer $\mathrm{MoS}_{2}$, graphene, and graphite are summarized. For comparison, we performed similar temperature-dependent Raman measurements on sapphire-supported monolayer $\mathrm{MoS}_{2}$ (Table 1 and Supporting Information) and found no significant difference in the first-order temperature coefficients for either mode. The small difference between $\chi_{T}$ coefficients for the $E_{2 g}^{1}$ mode in suspended versus sapphire-supported likely results from varied in-plane strain applied by the substrate since $E_{2 g}^{1}$ mode is prone to strain in $\mathrm{MoS}_{2}$ while $A_{1 g}$ is not. ${ }^{19}$ As a result, the temperature-dependent phonon frequency shift determined in this study can be used to monitor local temperature evolution in the $\mathrm{MoS}_{2}$ nanostructure by recording the change of $A_{1 g}$ peak under various external modifications, as was demonstrated with graphene. ${ }^{30}$

Strong thermal effects induced by the excitation laser power have been observed in the Raman spectra from substrate-supported $\mathrm{MoS}_{2}$ flakes. ${ }^{17,20}$ It is likely that the substrate plays an important role in heat dissipation; therefore, the previously reported behavior might not be intrinsic to $\mathrm{MoS}_{2}$. To obtain a comprehensive understanding of the intrinsic $\mathrm{MoS}_{2}$ property, we further studied the laser-power-dependent Raman spectra on suspended flake. These Raman

TABLE 1. First-Order Temperature Coefficients $\chi_{T}$, Power Coefficients $\chi_{P}$, and Thermal Conductivity $\kappa$ Obtained from Raman Spectra ${ }^{a}$

\begin{tabular}{|c|c|c|c|c|c|}
\hline material & type & mode & $\chi_{T}\left(\left(\mathrm{~cm}^{-1}\right) /(\mathrm{K})\right)$ & $\chi_{P}\left(\left(\mathrm{~cm}^{-1}\right) /(\mathrm{mW})\right)$ & $\kappa((\mathrm{W}) /(\mathrm{mK}))$ \\
\hline \multirow[t]{2}{*}{$\mathrm{MoS}_{2}$ monolayer } & suspended & $E_{2 g}^{1}$ & -0.011 & -12.8 & \\
\hline & & $A_{1 g}$ & -0.013 & -10.9 & 34.5 \\
\hline \multirow[t]{2}{*}{$\mathrm{MoS}_{2}$ monolayer } & $\mathrm{Si}_{3} \mathrm{~N}_{4}$-supported & $E_{2 g}^{1}$ & & -12.9 & \\
\hline & & $A_{1 g}$ & & -10.0 & \\
\hline \multirow[t]{2}{*}{$\mathrm{MoS}_{2}$ monolayer } & sapphire-supported & $E_{2 g}^{1}$ & -0.017 & $-0.09^{c}$ & \\
\hline & & $A_{1 g}$ & -0.013 & $-2.2^{c}$ & \\
\hline \multirow[t]{2}{*}{$\mathrm{MoS}_{2}$ few-layer } & suspended & $E_{2 g}^{1}$ & $-0.013^{b}$ & $-5.7^{b}$ & $52^{b}$ \\
\hline & & $A_{1 g}$ & $-0.012^{b}$ & $-5.4^{b}$ & \\
\hline graphene monolayer & suspended & $G$ & & $-1.2^{d}$ & $5300 \pm 480^{d}$ \\
\hline graphene monolayer & supported & $G$ & $-0.016^{e}$ & & \\
\hline graphene bilayer & supported & $G$ & $-0.015^{f}$ & & \\
\hline HOPG & & $G$ & $-0.011^{g}$ & & \\
\hline
\end{tabular}

${ }^{a}$ Bold entries represent data from our group including this work and ref 17. ${ }^{b}$ ref 27. ${ }^{c}$ ref $17 .{ }^{d}$ ref $26 .{ }^{e}$ ref 25. ${ }^{f}$ ref $9 .{ }^{g}$ ref 31. 
spectra are collected in a WITec Raman spectrometer using a $488 \mathrm{~nm}$ solid-state laser for excitation with the beam focused by a $100 \times$ objective lens (NA $=0.9$ ). The estimated Gaussian beam width is approximately $0.34 \mu \mathrm{m}$. Here the Raman system with a high NA is used to ensure the laser beam is smaller than the hole diameter. Detailed analysis (shown in the later section) confirms that the size of the holes is sufficiently large to allow for extraction of the monolayer $\mathrm{MoS}_{2}$ thermal conductivity with high accuracy, though its diameter is only about twice larger than the laser beam spot size. It is because of the low thermal conductivity of monolayer $\mathrm{MoS}_{2}$. On the other hand, to measure flakes with high thermal conductivity such as graphene or BN, it is necessary to employ much larger holes.

Four representative room-temperature Raman spectra collected at laser powers spanning from 0.040 to $0.164 \mathrm{~mW}$ are shown in Figure 3(a). As the laser power increases, both of the Raman-active modes soften because of local heating of the monolayer $\mathrm{MoS}_{2}$. To avoid damage to the sample and to stay within the linear dependence range, we kept the excitation laser power below $0.25 \mathrm{~mW}$. The Lorentzian-fit Raman peak frequencies as a function of incident laser power are plotted in Figure $3(\mathrm{~b})$, where both the $E_{2 g}^{1}$ and $A_{1 g}$ modes soften linearly with increasing power. Beyond $0.25 \mathrm{~mW}$ of laser power, this thermal behavior saturates (see Figure S3, Supporting Information). The appearance of nonlinear effects results either from the nonlinearity of absorption or higher orders of the temperature-dependent coefficients. In Figure 3(b), we show the power-dependent peak positions in the linear, low-power range, which is characterized by

$$
\Delta \omega=\omega\left(P_{2}\right)-\omega\left(P_{1}\right)=\chi_{P}\left(P_{2}-P_{1}\right)=\chi_{P} \Delta P
$$

Here $\chi_{P}$, the slope of power dependence in the linear region, is the first-order power-dependent coefficient and $P$ is the laser power. As with $\chi_{T}$, the fitted coefficients $\chi_{P}$ for the $E_{2 g}^{1}$ and $A_{1 g}$ modes are very similar, $-(12.8 \pm 0.2)$ and $-(10.9 \pm 0.4) \mathrm{cm}^{-1} / \mathrm{mW}$, respectively. Note that these $\chi_{P}$ values significantly exceed those reported ${ }^{17}$ for sapphire-supported monolayer $\mathrm{MoS}_{2}$ and exceed by a factor of 2 those for suspended few-layer flakes. ${ }^{27}$ In Table 1, we also summarize the laser power coefficients for various $\mathrm{MoS}_{2}$ flakes. For suspended monolayer $\mathrm{MoS}_{2}$, both modes behave similarly in terms of the power-dependent slope, while for the sapphire-supported monolayer flake, the in-plane $E_{2 g}^{1}$ mode shows much smaller laser power dependence compared to the out-of-plane $A_{1 g}$ mode. ${ }^{17}$ This is a strong manifestation of strain in $\mathrm{MoS}_{2}$. Strain is induced since the expansion of the $\mathrm{MoS}_{2}$ lattice due to laser heating is hindered by the cooler sapphire substrate (near RT, by assuming negligible absorption of the laser by the substrate). This point is further supported by a recent study on strain-dependent Raman spectra of monolayer $\mathrm{MoS}_{2}$ where only the $E_{2 g}^{1}$ mode is sensitive (a)

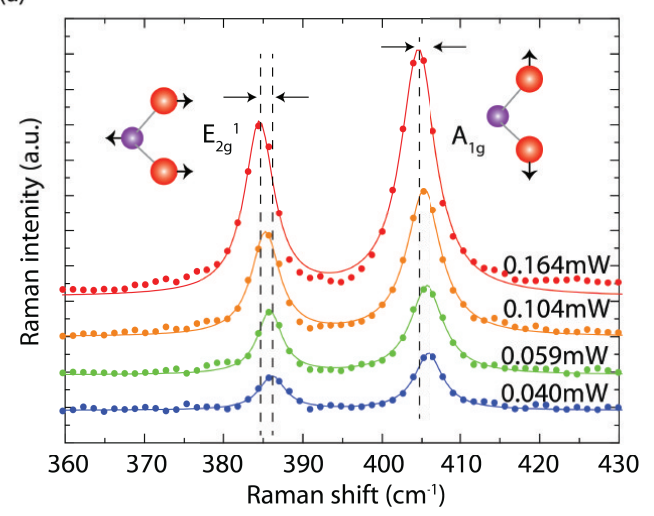

(b)

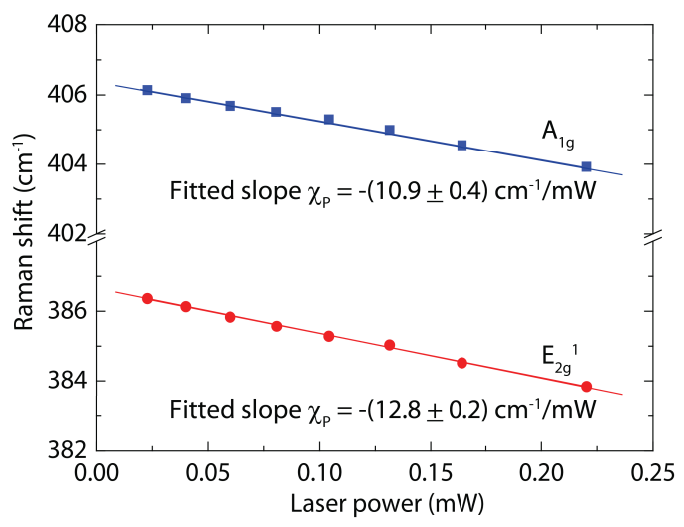

Figure 3. (a) Four example Raman spectra of suspended, monolayer $\mathrm{MoS}_{2}$ at increasing excitation laser power at RT in air environment. Spectra offset vertically for clarity. (b) Raman peak frequencies for $A_{1 g}$ (blue squares) and $E_{2 g}^{1}$ (red circles) modes as a function of laser power. Fit lines and resulting linear power coefficients $\chi_{P}$ are shown.

to the strain variation, but the $A_{1 g}$ mode does not respond. ${ }^{19}$

Taking advantage of the sensitive response of phonon frequency in $\mathrm{MoS}_{2}$ to local heating by the laser, we are able to estimate the thermal conductivity of this $2 \mathrm{D}$ material in a noncontact method. Although the Ramanactive optical phonons do not play a significant role in directly conducting heat along the flake, their frequency of vibration is reasonably sensitive to the local temperature fluctuations caused by external effects. The temperature increase of the suspended flake under laser excitation is directly related to the thermal conductivity of the material, assuming that the absorbed heat transfers radially through a small crosssectional area of the monolayer flake from the center to the edge. The cross section of the substrate and schematic of heat flow is shown in Figure 4(a). Therefore, inside the hole region, we can write the heat dissipation equation ignoring the heat conducted to the air as

$$
\kappa \frac{1}{r} \frac{\mathrm{d}}{\mathrm{d} r}\left[r \frac{\mathrm{d} T_{1}(r)}{\mathrm{d} r}\right]+q(r)=0 \text { for } r<R
$$

where $T_{1}(r)$ is the temperature distribution inside the hole, $r$ is radial position from the center of the 


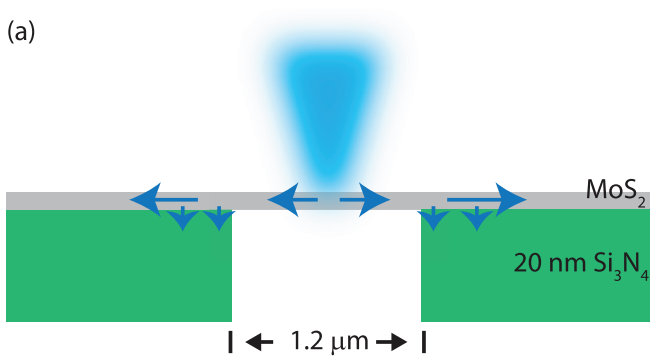

(b)

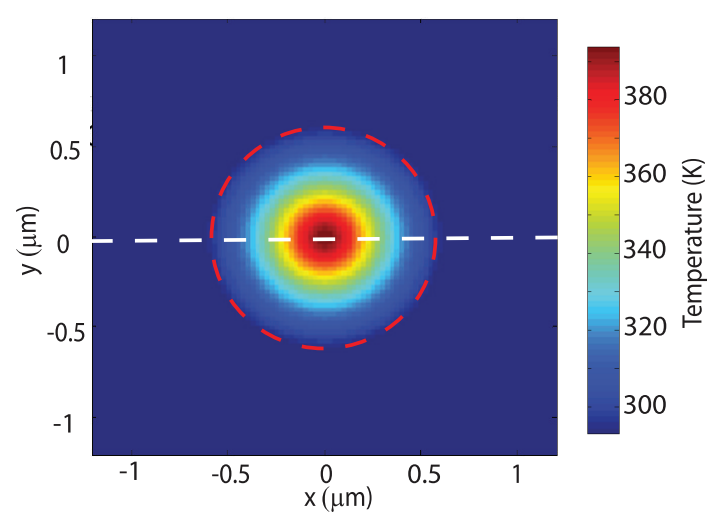

(c)

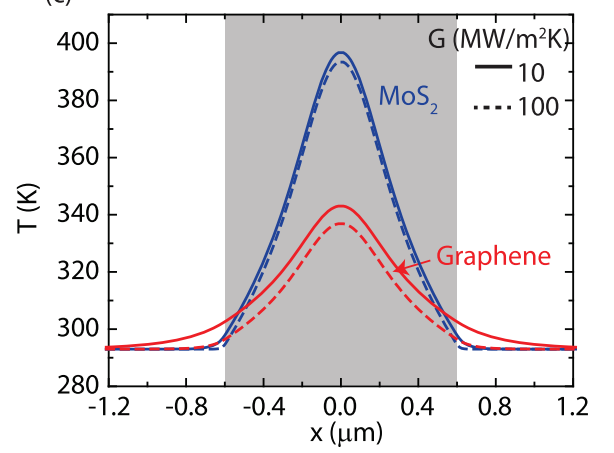

Figure 4. (a) Cross section of the sample structure and schematic of the heat flow in monolayer $\mathrm{MoS}_{2}$ suspended over a hole in $20 \mathrm{~nm}$ thick $\mathrm{Si}_{3} \mathrm{~N}_{4}$ under laser illumination. (b) Calculated temperature profile of the suspended $\mathrm{MoS}_{2}$ under a $0.1 \mathrm{~mW}$ laser excitation by assuming $G=50 \mathrm{MW} / \mathrm{m}^{2} \mathrm{~K}$, and $\kappa=34.5 \mathrm{~W} / \mathrm{mK}$. The temperature at the center of the hole reaches about $390 \mathrm{~K}$ but drops to $295 \mathrm{~K}$ at the edge of the hole because of the low thermal conductivity of $\mathrm{MoS}_{2}$. The red dashed circle marks the region of suspended monolayer $\mathrm{MoS}_{2}$. (c) Calculated temperature profile along the $x$-axis at $y=0$ for both graphene (red, at $5 \mathrm{~mW}$ laser power) and $\mathrm{MoS}_{2}$ (blue, at $0.1 \mathrm{~mW}$ laser) assuming in plan thermal conductivity of graphene and $\mathrm{MoS}_{2}$ are respectively $2000 \mathrm{~W} / \mathrm{mK}^{31}$ and 34.5 $\mathrm{W} / \mathrm{mK}$. The temperature distribution of graphene is highly sensitive to the interfacial conductance $G$, while that of $\mathrm{MoS}_{2}$ is not, because of the low thermal conductivity of $\mathrm{MoS}_{2}$.

hole, $R=0.6 \mu \mathrm{m}$ is the radius of the hole, and $\kappa$ is the thermal conductivity of the suspended $\mathrm{MoS}_{2}$. In the above equation, $q(r)$ is volumetric Gaussian beam heating and given $\mathrm{as}^{28}$

$$
q(r)=\frac{l \alpha}{t} \exp \left(-\frac{r^{2}}{r_{0}^{2}}\right)
$$

where $\alpha=(9 \pm 1) \%$ is the absorbance of monolayer $\mathrm{MoS}_{2}$ at $488 \mathrm{~nm}$, which is extrapolated from Figure 4(a) in ref $8, t=0.65 \mathrm{~nm}$ is the thickness of the flake, $r_{0}=0.17 \mu \mathrm{m}$ is the half of the Gaussian beam width, $I=P /\left(\pi r_{0}{ }^{2}\right)$ is the peak laser power per unit area at the center of beam spot. Outside the hole, the heat transports not only along the flake, but also into the $\mathrm{Si}_{3} \mathrm{~N}_{4}$ substrate, so its heat dissipation can be described as

$$
\kappa^{\prime} \frac{1}{r} \frac{\mathrm{d}}{\mathrm{d} r}\left[r \frac{\mathrm{d} T_{2}(r)}{\mathrm{d} r}\right]-\frac{G}{t}\left[\left(T_{2}(r)-T_{a}\right)\right]=0 \text { for } r>R
$$

where $T_{2}(r)$ is the temperature profile outside the hole, $G$ is the interfacial thermal conductance bewteen $\mathrm{MoS}_{2}$ and $\mathrm{Si}_{3} \mathrm{~N}_{4}$ substrate, $T_{a}$ is the ambient temperature, and $\kappa^{\prime}$ is the thermal conductivity of the supported $\mathrm{MoS}_{2}$. The solutions to eqs 3 and 5 are

$$
\begin{gathered}
T_{1}(r)=c_{1}+c_{2} \ln (r)+c_{3} E i\left(-\frac{r^{2}}{r_{0}^{2}}\right) \\
T_{2}(\gamma)=c_{4} I_{0}(\gamma)+c_{5} K_{0}(\gamma)+T_{a}
\end{gathered}
$$

where $c_{i}$ are constants to be determined by boundary conditions, $I_{0}$ and $K_{0}$ are the zero-order modified bessel function of the first and second kind, respectively, $\gamma=\left(G /\left(\kappa^{\prime} t\right)\right)^{1 / 2}$, and $E i(x)=-\int_{-x}^{\infty} e^{-u} / u \mathrm{~d} t$ is the exponential integral. With suitable boundary conditions (see Supporting Information), the temperature distribution $T_{1}(r)$ and $T_{2}(r)$ under various laser powers can be solved with three unknown parameters $\kappa, \kappa^{\prime}$, and $G$.

On the other hand, the weighted average temperature measured by Raman spectroscopy inside the beam spot is ${ }^{28,32}$

$$
T_{m} \approx \frac{\int_{0}^{R} T_{1}(r) q(r) r d r}{\int_{0}^{R} q(r) r d r}
$$

By fitting this model with the $T_{m} v s P$ determined by the Raman measurements, one can extract the thermal conductivity $\kappa$ of suspended $\mathrm{MoS}_{2}$ given the values of $\kappa^{\prime}$ and G. Here the parameters extracted on mode are used since it is less affected by strain. ${ }^{19}$ If we make an assumption that the supported and suspended $\mathrm{MoS}_{2}$ have the same thermal conductivity $\left(\kappa=\kappa^{\prime}\right)$ and $G=50 \mathrm{MW} / \mathrm{m}^{2} \mathrm{~K}$, a typical value for van der Waals interfaces, ${ }^{33}$ the thermal conductivity value is extracted to be $34.5 \mathrm{~W} / \mathrm{mK}$. In fact, it is found that for a value of $G$ ranging from 10 to $300 \mathrm{MW} / \mathrm{m}^{2} \mathrm{~K}$, the extracted $\kappa$ slightly changes by $\pm 0.4 \mathrm{~W} / \mathrm{mK}$ only, suggesting that the interface is not the dominant factor for thermal resistance. Also if we set $\kappa^{\prime}=0.1 \kappa$ by taking the reduction of thermal conductivity due to substrate interaction into consideration, the fitted $\kappa$ only increased slightly to be $35.3 \mathrm{~W} / \mathrm{mK}$. That is to say, values of both $\kappa^{\prime}$ and $G$ affect $\kappa$ by less than $1 \mathrm{~W} / \mathrm{mK}$. This could be attributed to the relatively low thermal conductivity 
of $\mathrm{MoS}_{2}$. Including the uncertainty of the Raman shifts from measurement setups $\left( \pm 1 \mathrm{~cm}^{-1}\right)$, as well as errors of fitted power- and temperature-dependent linear coefficients, $^{31}$ the extracted thermal conductivity is $\kappa=(34.5 \pm 4) \mathrm{W} / \mathrm{mK}$ at RT. This low thermal conductivity value is very close to what was recently reported ${ }^{19,27}$ for few-layer flakes $\mathrm{MoS}_{2} \approx 52 \mathrm{~W} / \mathrm{mK}$ and 2 orders of magnitude less than that of monolayer graphene. ${ }^{26}$ Besides the uncertainty due to measurement setup and data analysis, the accuracy of the extracted thermal conductivity could be also affected by any remaining PMMA residues on the sample surface, air surrounding the sample instead of a vacuum, ${ }^{34}$ etc., which need to be scrutinized in future work. It is worth noting that the power dissipation through PL excited by $488 \mathrm{~nm}$ laser is negligible because of the low PL quantum yield $\left(\sim 4 \times 10^{-3}\right)$ of monolayer $\mathrm{MoS}_{2} .{ }^{8}$

To justify that interfacial conductance and thermal conductivity of the supported portion of the flake are not playing a significant role in determination of the thermal conductivity, we calculated the temperature profile of suspended monolayer $\mathrm{MoS}_{2}$ if the heat source is placed on the suspended region, as shown in Figure 4(b). To satisfy the experimentally observed high temperature at the center of the hole $(\Delta T \sim 100 \mathrm{~K})$, the extracted thermal conductivity of suspended $\mathrm{MoS}_{2}$ has to be low. Moreover, the profiles shown in Figure 4 confirm that the $1.2 \mu \mathrm{m}$ diameter of the hole used in this study, albeit small, is sufficiently large to extract the $\mathrm{MoS}_{2}$ thermal conductivity with high accuracy. Because of the low thermal conductivity of the suspended $\mathrm{MoS}_{2}$, the temperature drop is steep from the center of the hole to the edge. At the edge, the $\mathrm{MoS}_{2}$ temperature is nearly at the ambient temperature; as a result, the extracted thermal conductivity of suspended $\mathrm{MoS}_{2}$ is practically independent of the thermal conductivity of the supported $\mathrm{MoS}_{2}$ or the interface thermal conductivity between $\mathrm{MoS}_{2}$ and $\mathrm{Si}_{3} \mathrm{~N}_{4}$. To further illustrate the difference between $\mathrm{MoS}_{2}$ and the already widely studied graphene, we also calculated the temperature profiles of monolayer $\mathrm{MoS}_{2}$ and graphene suspended over $1.2 \mu \mathrm{m}$ holes, respectively, which are plotted in Figure $4(\mathrm{c})$. Since graphene has a lower absorption coefficient $(2.3 \%)^{35}$ and an extraordinarily high in-plane thermal conductivity ( $\kappa$ (graphene) $=2000 \mathrm{~W} / \mathrm{mK}$ ), ${ }^{31}$ a much higher laser power of $5 \mathrm{~mW}$ was assumed in the calculation, $50 \times$ that for $\mathrm{MoS}_{2}$, to induce appreciable temperature changes in graphene. It is observed that for single layer graphene suspended over a small hole, its temperature profile depends sensitively on $G$, confirming the necessity to employ large holes for studying materials with high thermal conductivity such as graphene.

We also conducted a first-principles lattice dynamics simulation to calculate the thermal conductivity of monolayer suspended $\mathrm{MoS}_{2}$. The lattice thermal conductivity $\kappa$ of a 2D crystal can be calculated using the phonon Boltzmann transport equation under the single mode relaxation time approximation giving

$$
\kappa=\frac{1}{V N_{k}} \sum_{k, \lambda} c_{k, \lambda} v_{k, \lambda}^{2} \tau_{k, \lambda}
$$

where the subscript $k$ refers to the wave-vector in the first Brillouin zone, $\lambda$ refers to different phonon branches, $V$ is the volume of the primitive unit cell with its thickness set to be the same with the interlayer distance in bulk $\operatorname{MoS}_{2}(0.65 \mathrm{~nm}), N_{k}$ is the number of discrete $k$-points, $c$ denotes the heat capacity per mode derived from the Bose-Einstein distribution, $v$ the group velocity, $\tau$ the phonon relaxation time, and $\Lambda=v \tau$ the phonon mean free path. To evaluate the thermal conductivity, we obtained the heat capacity per mode and the group velocity from harmonic properties of the phonons (phonon dispersion relation) which are determined by the harmonic force constants. The relaxation times due to intrinsic, threephonon scattering are calculated by Fermi's golden rule using the anharmonic force constants. ${ }^{36}$ Higher order scattering terms are not included since their effects are typically much smaller than those from the third order scattering. ${ }^{37}$ The force constants are calculated using a finite difference method with the forces computed from first-principles density functional theory calculations. (See Supporting Information for further information.)

Besides three-phonon anharmonic scattering, boundary scattering also affects phonon relaxation times. Therefore the total relaxation time can be calculated using the Matthiessen's rule

$$
\tau^{-1}=\tau_{\mathrm{ph}-\mathrm{ph}}^{-1}+\frac{v}{l}
$$

where the second term describes boundary scattering rate and $/$, the limiting length for boundary scattering, is dimension of the material. In our calculation, the diameter of the hole $(1.2 \mu \mathrm{m})$ is used for the limiting length to match the experimental condition. The calculated thermal conductivity is $35.5 \mathrm{~W} / \mathrm{mK}$ at RT. This model closely reproduces the experimental value; however, it does not yet account for possible phonon scattering by defects such as PMMA residue, sample oxidation, and other defects produced during sample fabrication. All of such defects can scatter phonons and reduce thermal conductivity. To further understand whether the relatively small hole size in $\mathrm{Si}_{3} \mathrm{~N}_{4}$ used in this work adversely impacts the measurement of $\kappa$, we also calculated $\kappa$ as a function of boundary scattering length / (Figure S7, Supporting Information). As expected, $\kappa$ increases with increasing $/$, but for $/>1.2 \mu \mathrm{m}$, the increase in $\kappa$ becomes negligible. This observation is expected since the calculated three-phonon anharmonic scattering mean free path is much smaller than $1.2 \mu \mathrm{m}$. This provides another independent evidence, in addition to what is presented in Figure 4, that a hole 
size of $1.2 \mu \mathrm{m}$ employed in this work, albeit small, is sufficient in extracting the $\kappa$ value of monolayer $\mathrm{MoS}_{2}$.

It is well-known that both Mo and $S$ have rich isotopes. In this study, we used the weighted mass of naturally occurring Mo and $\mathrm{S}$. It is possible that the thermal conductivity in isotopically pure $\mathrm{MoS}_{2}$ is much higher than that in naturally occurring $\mathrm{MoS}_{2}$. It is also interesting to note that the thermal conductivity value of exfoliated monolayer $\mathrm{MoS}_{2}$ extracted in this study, $\sim 34.5 \mathrm{~W} / \mathrm{mK}$, is smaller than that reported for the 11layer $\mathrm{MoS}_{2}$ prepared by chemical vapor deposition, $\sim 52 \mathrm{~W} / \mathrm{mK}^{27}$ This apparent trend is opposite to that in graphene: the monolayer graphene possesses a higher thermal conductivity than few-layer graphene because of suppressed Umklapp scattering in monolayer graphene. However, the direct comparison between these two results is probably invalid since the samples were prepared differently and the sample quality might not be comparable. To understand thermal conductivity in 2D $\mathrm{MoS}_{2}$ more work in both experiments and theory is necessary. Nevertheless, in this study we are able to provide a useful estimate of thermal conductivity of exfoliated monolayer $\mathrm{MoS}_{2}$; its value is low, especially compared to that of graphene, which largely results from its low group velocities of acoustic phonons due to the large volume and large mass of the $\mathrm{MoS}_{2}$ primitive unit cell.

\section{CONCLUSIONS}

In conclusion, temperature-dependent Raman spectra of suspended, monolayer $\mathrm{MoS}_{2}$ are reported for the first time. Both $E_{2 g}^{1}$ and $A_{1 g}$ modes soften linearly as the temperature increases, with first-order linear coefficients of $0.011 \mathrm{~cm}^{-1} / \mathrm{K}$ and $0.013 \mathrm{~cm}^{-1} / \mathrm{K}$, respectively. Sample temperature and laser power dependence are combined and compared to those of other relevant 2D materials. Suspended and substrate-supported monolayer flakes exhibit similar $A_{1 g}$ mode behavior, with differences observed in the $E_{2 g}^{1}$ mode resulting from the presence of substrate-induced strain. The extracted thermal conductivity of suspended monolayer $\mathrm{MoS}_{2}$ at RT is $\kappa=(34.5 \pm 4) \mathrm{W} / \mathrm{mK}$ at RT. A value close to the experimentally observed thermal conductivity has been estimated by a first-principles model. This work provides important information about the thermal properties of monolayer, which will facilitate future electronic and optical applications of $\mathrm{MoS}_{2}$.

\section{METHODS}

Monolayer $\mathrm{MoS}_{2}$ was exfoliated from naturally occurring bulk $\mathrm{MoS}_{2}$ and deposited onto a Si substrate covered with $270 \mathrm{~nm}$ $\mathrm{SiO}_{2}$, on top of which polyvinyl alcohol (PVA) and polymethyl methacrylate (PMMA) films were spin-coated. ${ }^{38,39}$ Once AFM identified a monolayer flake, the sample was immersed in water to dissolve the PVA film. The PMMA layer with target flake attached then floated on the water. Next a glass slide was used to extract the film and facilitate transfer to the previously patterned $\mathrm{Si}_{3} \mathrm{~N}_{4} / \mathrm{SiO}_{2} / \mathrm{Si}$ substrate as shown schematically in Figure 1 (c). Finally, the sample was kept in a vacuum at $400{ }^{\circ} \mathrm{C}$ for $4 \mathrm{~h}$ to remove the PMMA film.

Conflict of Interest: The authors declare no competing financial interest.

Acknowledgment. The authors acknowledge the support from NSF (CAREER ECCS-084910, ECCS-1232191, monitored by Anupama Kaul), AFOSR (FA9550-12-1-0257, monitored by James Hwang), NIST (60NANB12D147, monitored by David Gundlach), the Notre Dame Center for Research Computing and NSF through TeraGrid resources provided by SDSC Trestles (Grant Number TG-CTS100078), the Center for Nanoscience and Technology (NDnano) at the University of Notre Dame, Swiss National Science Foundation (Grants 132102 and 138237), and the Swiss Nanoscience Institute (NCCR Nanoscience).

Supporting Information Available: Supporting figures and calculations. This material is available free of charge via the Internet at http://pubs.acs.org.

\section{REFERENCES AND NOTES}

1. Novoselov, K. S.; Geim, A. K.; Morozov, S. V.; Jiang, D.; Katsnelson, M. I.; Grigorieva, I. V.; Dubonos, S. V.; Firsov, A. A. Two-Dimensional Gas of Massless Dirac Fermions in Graphene. Nature 2005, 438, 197-200.

2. Novoselov, K. S.; Jiang, D.; Schedin, F.; Booth, T. J.; Khotkevich, V. V.; Morozov, S. V.; Geim, A. K. Two-Dimensional Atomic Crystals. Proc. Natl. Acad. Sci. U. S. A. 2005, 102, 1045110453.

3. Geim, A. K.; Novoselov, K. S. The Rise of Graphene. Nat. Mater. 2007, 6, 183-191.

4. Wang, Q. H.; Kalantar-Zadeh, K.; Kis, A.; Coleman, J. N.; Strano, M. S. Electronics and Optoelectronics of TwoDimensional Transition Metal Dichalcogenides. Nat. Nanotechnol. 2012, 7, 699-712.

5. Hwang, W. S.; Remskar, M.; Yan, R.; Protasenko, V.; Tahy, K.; Chae, S. D.; Zhao, P.; Konar, A.; Xing, H. G.; Seabaugh, A.; et al. Transistors with Chemically Synthesized Layered Semiconductor $\mathrm{WS}_{2}$ Exhibiting $10^{5}$ Room Temperature Modulation and Ambipolar Behavior. Appl. Phys. Lett. 2012, 101, 013107.

6. Hwang, W. S.; Remskar, M.; Yan, R.; Kosel, T.; Park, J. K.; Cho, B. J.; Haensch, W.; Xing, H. G.; Seabaugh, A.; Jena, D. Comparative Study of Chemically Synthesized and Exfoliated Multilayer $\mathrm{MoS}_{2}$ Field-Effect Transistors. Appl. Phys. Lett. 2013, 102, 043116.

7. Splendiani, A.; Sun, L.; Zhang, Y.; Li, T.; Kim, J.; Chim, C.-Y.; Galli, G.; Wang, F. Emerging Photoluminescence in Monolayer $\mathrm{MoS}_{2}$. Nano Lett. 2010, 10, 1271-1275.

8. Mak, K. F.; Lee, C.; Hone, J.; Shan, J.; Heinz, T. F. Atomically Thin $\mathrm{MoS}_{2}$ : A New Direct-Gap Semiconductor. Phys. Rev. Lett. 2010, 105, 136805.

9. Gutiérrez, H. R.; Perea-López, N.; Elas, A. L.; Berkdemir, A.; Wang, B.; Lv, R.; López-Uras, F.; Crespi, V. H.; Terrones, H.; Terrones, M. Extraordinary Room-Temperature Photoluminescence in Triangular $\mathrm{WS}_{2}$ Monolayers. Nano Lett. 2012, 13, 3447.

10. Radisavljevic, B.; Radenovic, A.; Brivio, J.; Giacometti, V.; Kis, A. Single-layer $\mathrm{MoS}_{2}$ Transistors. Nat. Nanotechnol. 2011, 6, 147-150.

11. Kim, S.; Konar, A.; Hwang, W.-S.; Lee, J. H.; Lee, J.; Yang, J.; Jung, C.; Kim, H.; Yoo, J.-B.; Choi, J. Y.; et al. High-mobility and Low-Power Thin-Film Transistors Based on Multilayer $\mathrm{MoS}_{2}$ Crystals. Nat. Commun. 2012, 3, 1011.

12. Han, S. W.; Kwon, H.; Kim, S. K.; Ryu, S.; Yun, W. S.; Kim, D. H.; Hwang, J. H.; Kang, J.-S.; Baik, J.; Shin, H. J.; et al. Band-Gap Transition Induced by Interlayer Van der Waals Interaction in $\mathrm{MoS}_{2}$. Phys. Rev. B: Condens. Matter Mater. Phys. 2011, $84,045409$. 
13. Komsa, H.-P.; Krasheninnikov, A. V. Effects of Confinement and Environment on the Electronic Structure and Exciton Binding Energy of $\mathrm{MoS}_{2}$ from First Principles. Phys. Rev. B: Condens. Matter Mater. Phys. 2012, 86, 241201.

14. Lee, C.; Yan, H.; Brus, L. E.; Heinz, T. F.; Hone, J.; Ryu, S. Anomalous Lattice Vibrations of Single- and Few-Layer $\mathrm{MoS}_{2}$. ACS Nano 2010, 4, 2695-2700.

15. Ferrari, A. C.; Meyer, J. C.; Scardaci, V.; Casiraghi, C.; Lazzeri, M.; Mauri, F.; Piscanec, S.; Jiang, D.; Novoselov, K. S.; Roth, S.; et al. Raman Spectrum of Graphene and Graphene Layers. Phys. Rev. Lett. 2006, 97, 187401.

16. Berkdemir, A.; Gutiérrez, H. R.; Botello-Méndez, A. R.; PereaLópez, N.; Elas, A. L.; Chia, C.-I.; Wang, B.; Crespi, V. H.; López-Uras, F.; Charlier, J.-C.; et al. Identification of Individual and Few Layers of $\mathrm{WS}_{2}$ Using Raman Spectroscopy. Sci. Rep. 2013, 3, 1755.

17. Yan, R.; Bertolazzi, S.; Brivio, J.; Fang, T.; Konar, A.; Birdwell, A. G.; Nguyen, N. V.; Kis, A.; Jena, D.; Xing, H. G. Raman and Photoluminescence Study of Dielectric and Thermal Effects on Atomically Thin $\mathrm{MoS}_{2}$. arXiv:1211.4136 (2012).

18. Chakraborty, B.; Bera, A.; Muthu, D. V. S.; Bhowmick, S.; Waghmare, U. V.; Sood, A. K. Symmetry-Dependent Phonon Renormalization in Monolayer $\mathrm{MoS}_{2}$ Transistor. Phys. Rev. B: Condens. Matter Mater. Phys. 2012, 85, 161403.

19. Wang, Y.; Cong, C.; Qiu, C.; Yu, T. Raman Spectroscopy Study of Lattice Vibration and Crystallographic Orientation of Monolayer $\mathrm{MoS}_{2}$ under Uniaxial Strain. Small 2013, 10.1002/smll.201202876.

20. Najmaei, S.; Liu, Z.; Ajayan, P. M.; Lou, J. Thermal Effects on the Characteristic Raman Spectrum of Molybdenum Disulfide of Varying Thicknesses. Appl. Phys. Lett. 2012, 100, 013106.

21. Cowley, R. The Lattice Dynamics of an Anharmonic Crystal. Adv. Phys. 1963, 12, 421-480.

22. Postmus, C.; Ferraro, J. R.; Mitra, S. S. Pressure Dependence of Infrared Eigenfrequencies of $\mathrm{KCl}$ and $\mathrm{KBr}$. Phys. Rev. 1968, 174, 983-987.

23. Hart, T. R.; Aggarwal, R. L.; Lax, B. Temperature Dependence of Raman Scattering in Silicon. Phys. Rev. B: Solid State 1970, 1, 638-642.

24. Liu, M. S.; Bursill, L. A.; Prawer, S.; Beserman, R. Temperature Dependence of The First-Order Raman Phonon Line of Diamond. Phys. Rev. B: Condens. Matter Mater. Phys. 2000, 61, 3391-3395.

25. Calizo, I.; Balandin, A. A.; Bao, W.; Miao, F.; Lau, C. N. Temperature Dependence of the Raman Spectra of Graphene and Graphene Multilayers. Nano Lett. 2007, 7, 2645-2649.

26. Balandin, A. A.; Ghosh, S.; Bao, W.; Calizo, I.; Teweldebrhan, D.; Miao, F.; Lau, C. N. Superior Thermal Conductivity of Single-Layer Graphene. Nano Lett. 2008, 8, 902-907.

27. Sahoo, S.; Gaur, A. P. S.; Ahmadi, M.; Guinel, M. J.-F.; Katiyar, R. S. Temperature-Dependent Raman Studies and Thermal Conductivity of Few-Layer $\mathrm{MoS}_{2}$. J. Phys. Chem. C 2013, 117, 9042-9047.

28. Cai, W.; Moore, A. L.; Zhu, Y.; Li, X.; Chen, S.; Shi, L.; Ruoff, R. S. Thermal Transport in Suspended and Supported Monolayer Graphene Grown by Chemical Vapor Deposition. Nano Lett. 2010, 10, 1645-1651.

29. Beechem, T.; Graham, S.; Kearney, S. P.; Phinney, L. M.; Serrano, J. R. Invited Article: Simultaneous Mapping of Temperature and Stress in Microdevices Using MicroRaman Spectroscopy. Rev. Sci. Instrum. 2007, 78, 061301.

30. Calizo, I.; Miao, F.; Bao, W.; Lau, C. N.; Balandin, A. A. Variable Temperature Raman Microscopy as a Nanometrology Tool for Graphene Layers and Graphene-Based Devices. Appl. Phys. Lett. 2007, 91, 071913.

31. Balandin, A. a. Thermal Properties of Graphene and Nanostructured Carbon Materials. Nat. Mater. 2011, 10, 569-581.

32. Lee, J.-U.; Yoon, D.; Kim, H.; Lee, S. W.; Cheong, H. Thermal Conductivity of Suspended Pristine Graphene Measured by Raman Spectroscopy. Phys. Rev. B: Condens. Matter Mater. Phys. 2011, 83, 081419.
33. Luo, T.; Chen, G. Nanoscale Heat Transfer-from Computation to Experiment. Phys. Chem. Chem. Phys. 2013, 15, 3389-3412.

34. Chen, S.; Moore, A. L.; Cai, W.; Suk, J. W.; An, J.; Mishra, C.; Amos, C.; Magnuson, C. W.; Kang, J.; Shi, L.; et al. Raman Measurements of Thermal Transport in Suspended Monolayer Graphene of Variable Sizes in Vacuum and Gaseous Environments. ACS Nano 2011, 5, 321-328.

35. Nair, R. R.; Blake, P.; Grigorenko, A. N.; Novoselov, K. S.; Booth, T. J.; Stauber, T.; Peres, N. M. R.; Geim, A. K. Fine Structure Constant Defines Visual Transparency of Graphene. Science 2008, 320, 1308.

36. Luo, T.; Garg, J.; Shiomi, J.; Esfarjani, K.; Chen, G. Gallium Arsenide Thermal Conductivity and Optical Phonon Relaxation Times from First-Principles Calculations. Europhys. Lett. 2013, 101, 16001.

37. Lindsay, L.; Broido, D. A.; Mingo, N. Flexural Phonons and Thermal Transport in Graphene. Phys. Rev. B: Condens. Matter Mater. Phys. 2010, 82, 115427.

38. Bertolazzi, S.; Brivio, J.; Kis, A. Stretching and Breaking of Ultrathin $\mathrm{MoS}_{2}$. ACS Nano 2011, 5, 9703-9709.

39. Shi, H.; Yan, R.; Bertolazzi, S.; Brivio, J.; Gao, B.; Kis, A.; Jena, D.; Xing, H. G.; Huang, L. Exciton Dynamics in Suspended 2D Crystals. ACS Nano 2013, 7, 1072-1080. 